\title{
A UNIFIED APPROACH TO THE SUMMATION AND INTEGRATION FORMULAS FOR $q$-HYPERGEOMETRIC FUNCTIONS III
}

\author{
Mizan Rahman and Sergě̌ K. Suslov
}

\begin{abstract}
As a continuation of two previous reports, the summation formula for the bilateral very-well-poised ${ }_{6} \psi_{6}$ series is obtained from a Pearson-type difference equation on a symmetric $q$-quadratic lattice without the benefit of any transformation formula or other summation formulas. This formula then is used to obtain a formula for the very-well-poised, balanced ${ }_{8} \psi_{8}$ series which has the nonterminating $q$-Jackson formula and Gosper's bilateral Jackson formula as special cases. The corresponding Ramanujan- and Barnes-type integrals also are considered.
\end{abstract}

\section{Introduction}

This is the last in a series of papers (see [11] and [12]) in which the authors have addressed the question of how to derive the summation formulas for bilateral basic hypergeometric series as well as the corresponding formulas for the Ramanujan- and Barnes-type integrals (see also [13] and [14]) without the benefit of a single transformation formula. The basic ingredient of this approach is a Pearson-type difference equation of order one (see, for example, (1.1) of [12]). The coefficient functions $\sigma(s)$ and $\tau(s)$ are assumed to be polynomials of degrees at most 2 and 1, respectively, although in a latter section we will allow them to have a simple pole as well.

In [11] and [12] we were concerned only with the $q$-linear lattice arising out of the case $C_{1} C_{2}=0$ and exploited the parameters in $\sigma(s)$ and $\tau(s)$ to derive homogeneous or nonhomogeneous 2-term recurrence relations from (1.1) of [12] which, in turn, enabled us to obtain the well-known $q$-binomial formula, the ${ }_{1} \psi_{1}$ summation formula, the two summation formulas for the ${ }_{2} \phi_{1}$ series, the ${ }_{2} \psi_{2}$ summation formula as well as a formula for the ${ }_{3} \psi_{3}$ series. We also obtained the corresponding Ramanujan-type integration formula in each case and stated the Barnes-type integrals since they were derived elsewhere by essentially the same technique. The purpose of this paper is to complete the program by considering the $q$-quadratic lattice. In a sense, this is easier to deal with than the $q$-linear lattice because of the higher degree of symmetry that ensues. For example, if we denote $C_{2} / C_{1}=q^{\nu}, q \neq 1$, then

$$
x(s)=x(-s-\nu), \quad \nabla x_{1}(s)=C_{1} q^{-s-1 / 2}(1-q)\left(1-q^{\nu+2 s}\right),
$$

and the use of (1.5) of [12] gives

$$
\sigma(s)+\tau(s) \nabla x_{1}(s)=\sigma(-s-\nu) .
$$

Received November 15, 1997, revised August 27, 1988.

1991 Mathematics Subject Classification: 33D05, 33D15.

Key words and phrases: Pearson-type equation, symmetric $q$-quadratic lattice, ${ }_{6} \psi_{6}$ and $8 \psi_{8}$ summation formulas, nonterminating $q$-Jackson formula, Ramanujan- and Barnes- type integrals. 
The basic bilateral series ${ }_{r} \psi_{r}$ is an infinite series with $r$ numerator and $r$ denominator parameters

$$
{ }_{r} \psi_{r}\left[\begin{array}{c}
a_{1}, a_{2}, \ldots, a_{r} \\
b_{1}, b_{2}, \ldots, b_{r}
\end{array} ; q, z\right]=\sum_{n=-\infty}^{\infty} \frac{\left(a_{1}, a_{2}, \ldots, a_{r} ; q\right)_{n}}{\left(b_{1}, b_{2}, \ldots, b_{r} ; q\right)_{n}} z^{n}
$$

which converges absolutely in the annulus $\left|b_{1} b_{2} \cdots b_{r} / a_{1} a_{2} \cdots a_{r}\right|<|z|<1$. The series is called balanced if $z=q$ and $b_{1} b_{2} \cdots b_{r}=a_{1} a_{2} \cdots a_{r} q^{2}$. It is called well-poised if $a_{1} b_{1}=a_{2} b_{2}=\cdots=a_{r} b_{r}=q a$, say; it is called very-well-poised if, in addition, $b_{1}=a^{1 / 2}, b_{2}=-a^{1 / 2}$. In the latter case the series (1.3) contains a term $\left(1-a q^{2 n}\right)$ which, according to (1.1), represents a differential of the lattice $x(s)$. The argument $z$ is usually related to the parameters in a special way, particularly for those very-wellpoised series for which summation and transformation formulas exist. For further details, see [6]. We note that the bilateral series ${ }_{r} \psi_{r}$ can always be split into two unilateral ones:

$$
\begin{aligned}
{ }_{r} \psi_{r}\left[\begin{array}{c}
a_{1}, a_{2}, \ldots, a_{r} \\
b_{1}, b_{2}, \ldots, b_{r}
\end{array} ; q, z\right]= & \sum_{n=0}^{\infty} \frac{\left(a_{1}, a_{2}, \ldots, a_{r} ; q\right)_{n}}{\left(b_{1}, b_{2}, \ldots, b_{r} ; q\right)_{n}} z^{n} \\
& +\sum_{n=1}^{\infty} \frac{\left(q / b_{1}, q / b_{2}, \ldots, q / b_{r}\right)_{n}}{\left(q / a_{1}, q / a_{2}, \ldots, q / a_{r} ; q\right)_{n}}\left(\frac{b_{1} b_{2} \cdots b_{r}}{a_{1} a_{2} \cdots a_{r} z}\right)^{n}
\end{aligned}
$$

In Section 2, we consider a symmetric lattice $x(s)$ and polynomial-type coefficient functions in order to derive the well-known ${ }_{6} \psi_{6}$ summation formula of Bailey. The proof is very elementary and self-contained, in the sense that it doesn't use any transformation or other summation formulas of basic hypergeometric series. We believe that this is the simplest proof of the ${ }_{6} \psi_{6}$ formula. We also state the corresponding Ramanujan- and Barnes-type integrals that were proved elsewhere. In Section 3, we take the balanced rational function-type coefficient functions and derive a general formula for the very-well-poised and balanced ${ }_{8} \psi_{8}$ series, from which we deduce the nonterminating $q$-Jackson formula for the ${ }_{8} \phi_{7}$ series. Gosper's bilateral Jackson formula also emerges as a special case. Finally, we state the corresponding Ramanujanand Barnes- type integration formulas proved elsewhere by different authors.

2. The $q$-quadratic lattice $x(s)=\frac{1}{2}\left(q^{-s}+q^{s}\right)$ : the ${ }_{6} \psi_{6}$ summation formula

Starting with the simplest symmetric $q$-quadratic lattice $x(s)=\left(q^{-s}+q^{s}\right) / 2$, let us take

$$
\sigma(s)=q^{-2 s}\left(1-a q^{s-1}\right)\left(1-b q^{s-1}\right)\left(1-c q^{s-1}\right)\left(1-d q^{s-1}\right)
$$

where $a, b, c, d$ are arbitrary complex parameters. By (1.1) and (1.8), we have

$$
\frac{\rho(s+1)}{\rho(s)}=\frac{\sigma(-s)}{\sigma(s+1)}=q^{4 s+2} \frac{\left(1-a q^{-s-1}\right) \cdots\left(1-d q^{-s-1}\right)}{\left(1-a q^{s}\right) \cdots\left(1-d q^{s}\right)}
$$

with the general solution

$$
\rho(s)=\left(a q^{s}, a q^{-s}, b q^{s}, b q^{-s}, c q^{s}, c q^{-s}, d q^{s}, d q^{-s} ; q\right)_{\infty} q^{2 s^{2}} \omega(s)
$$

where $\omega(s \pm 1)=\omega(s)$. The choice of $\omega(s)$ is rather crucial in the evaluation of sums and integrals over $\rho(s)$. The choice is made in order that they converge, and the 
contributions from the boundaries are either zero or finite numbers. See [13] for the technique of choosing different $\omega(s)$ for Ramanujan- and Barnes-type integrals.

From (2.1) and (1.8), it follows that

$$
\begin{aligned}
\tau(s) \nabla x_{1}(s)= & \sigma(-s)-\sigma(s) \\
=[ & \left(1-a b / q^{2}\right)\left(1-a c / q^{2}\right)\left(1-a d / q^{2}\right) \\
& \left.\quad+\left(1-a b c d / q^{4}\right)\left(1-a q^{s-1}\right)\left(1-a q^{-s-1}\right)\right] \frac{q\left(q^{-s}-q^{s}\right)}{a},
\end{aligned}
$$

and hence, replacing $\rho(s)$ and $\omega(s)$ by $\rho(s ; a)$ and $\omega(s ; a)$, respectively (our intention being to set up a recurrence in the parameter $a)$, we obtain from (1.1):

$$
\begin{aligned}
\Delta[\rho(s ; a) \sigma(s)]=- & \frac{q}{a}\left(1-a b / q^{2}\right)\left(1-a c / q^{2}\right)\left(1-a d / q^{2}\right) \rho(s ; a)\left(q^{-s}-q^{s}\right) \\
& +\frac{q}{a}\left(1-a b c d / q^{4}\right) \frac{\omega(s ; a)}{\omega\left(s ; a q^{-1}\right)} \rho\left(s ; a q^{-1}\right)\left(q^{-s}-q^{s}\right) .
\end{aligned}
$$

Let $s_{0} \in \mathbf{C}$, such that $\operatorname{Im} s_{0} \neq 0$, and take

$$
\omega\left(s_{0} ; a\right)=\frac{q^{s_{0}-2 s_{0}^{2}}}{\left(a q^{s_{0}}, a q^{-s_{0}}, \ldots, d q^{s_{0}}, d q^{-s_{0}} ; q\right)_{\infty}\left(1-q^{2 s_{0}}\right)} .
$$

Denote $q^{s_{0}}=\alpha$, and

$$
\begin{aligned}
I_{\alpha}(a, b, c, d) & =\sum_{k=-\infty}^{\infty} \frac{(\alpha q / a, \alpha q / b, \alpha q / c, \alpha q / d ; q)_{k}}{(\alpha a, \alpha b, \alpha c, \alpha d ; q)_{k}} \frac{1-\alpha^{2} q^{2 k}}{1-\alpha^{2}}\left(\frac{a b c d}{q^{3}}\right)^{k} \\
& ={ }_{6} \psi_{6}\left[\begin{array}{c}
q \alpha,-q \alpha, \alpha q / a, \alpha q / b, \alpha q / c, \alpha q / d \\
\alpha,-\alpha, \alpha a, \alpha b, \alpha c, \alpha d
\end{array} ; q, a b c d / q^{3}\right] .
\end{aligned}
$$

Note that this very-well-poised bilateral series converges provided

$$
\left|\frac{a b c d}{q^{3}}\right|<1
$$

which we naturally shall assume to hold. Let us now sum (2.5) from $s=s_{0}-\ell$ to $s=s_{0}+k, \ell=1,2, \ldots, k=0,1,2, \ldots$, and then take the limits $\ell \rightarrow \infty$ and $k \rightarrow \infty$. This gives

$$
\begin{aligned}
& \left(1-a b c d q^{-4}\right)(1-\alpha a / q)(1-a / \alpha q) I_{\alpha}\left(a q^{-1}, b, c, d\right) \\
& \quad-\left(1-a b q^{-2}\left(1-a c q^{-2}\right)\left(1-a d q^{-2}\right) I_{\alpha}(a, b, c, d)\right. \\
& \quad=\frac{a q^{-1}}{\omega\left(s_{0}\right)}\left[\lim _{k \rightarrow \infty} \rho\left(s_{0}+k\right) \sigma\left(s_{0}+k\right)-\lim _{\ell \rightarrow \infty} \rho\left(s_{0}-\ell\right) \sigma\left(s_{0}-\ell\right)\right] .
\end{aligned}
$$

By (2.1), (2.3), and (2.6), we have

$$
\begin{aligned}
& \lim _{k \rightarrow \infty} \rho\left(s_{0}+k\right) \sigma\left(s_{0}+k\right) \\
&=\omega\left(s_{0}\right) q^{2 s_{0}^{2}-2 s_{0}}(a / \alpha, \alpha q / a, \ldots, d / \alpha, \alpha q / d ; q)_{\infty} \lim _{k \rightarrow \alpha}\left(\frac{a b c d}{q^{4}}\right)^{k}
\end{aligned}
$$


and

$$
\begin{aligned}
\lim _{\ell \rightarrow \infty} \rho\left(s_{0}-\ell\right) \sigma\left(s_{0}-\ell\right) & \\
& =\omega\left(s_{0}\right) q^{2 s_{0}^{2}-2 s_{0}}\left(\alpha a / q, q^{2} / \alpha a, \ldots, d \alpha / q, q^{2} / a \alpha ; q\right)_{\infty} \lim _{\ell \rightarrow \infty}\left(\frac{a b c d}{q^{4}}\right)^{\ell} .
\end{aligned}
$$

Thus the right-hand side of $(2.9)$ vanishes if $\left|a b c d / q^{4}\right|<1$. For the time being, we shall assume this to be true. By analytic continuation, we will be able to show that the final result is true under the less restrictive condition (2.8). Replacing $a$ by $a q$, we then obtain, from (2.9), the homogeneous recurrence formula

$$
I_{\alpha}(a, b, c, d)=\frac{(1-a b / q)(1-a c / q)(1-a d / q)}{(1-a \alpha)(1-a / \alpha)\left(1-a b c d / q^{3}\right)} I_{\alpha}(a q, b, c, d) .
$$

Iterating it $n-1$ times, $n=2,3, \ldots$, we get

$$
I_{\alpha}(a, b, c, d)=\frac{(a b / q, a c / q, a d / q ; q)_{n}}{\left(\alpha a, a / \alpha, a b c d / q^{3} ; q\right)_{n}} I_{\alpha}\left(a q^{n}, b, c, d\right) .
$$

Since

$$
\begin{aligned}
\lim _{n \rightarrow \infty} & I_{\alpha}\left(a q^{n}, b, c, d\right) \\
& =\lim _{n \rightarrow \infty} \sum_{k=-\infty}^{\infty} \frac{\left(1-\alpha^{2} q^{2 k}\right)\left(\alpha q^{1-n} / a, \alpha q / b, \alpha q / c, \alpha q / d ; q\right)_{k}}{\left(1-\alpha^{2}\right)\left(a \alpha q^{n}, b \alpha, c \alpha, d \alpha ; q\right)_{k}}\left(a b c d q^{n-3}\right)^{k} \\
& =\sum_{k=-\infty}^{\infty} \frac{\left(1-\alpha^{2} q^{2 k}\right)(\alpha q / b, \alpha q / c, \alpha q / d ; q)_{k}}{\left(1-\alpha^{2}\right)(b \alpha, c \alpha, d \alpha ; q)_{k}}\left(-\frac{\alpha b c d}{q^{2}}\right)^{k} q^{\left(\begin{array}{c}
k \\
2
\end{array}\right)}
\end{aligned}
$$

exists, we find that

$$
I_{\alpha}(a, b, c, d)=\frac{(a b / q, a c / q, a d / q ; q)_{\infty}}{\left(a \alpha, a / \alpha, a b c d / q^{3} ; q\right)_{\infty}} \lim _{n \rightarrow \infty} I_{\alpha}\left(a q^{n}, b, c, d\right) .
$$

However, the symmetry of $I_{\alpha}(a, b, c, d)$ in $a, b, c, d$ is obvious from its definition (2.7), so we deduce that

$$
I_{\alpha}(a, b, c, d)=\frac{(a b / q, a c / q, a d / q, b c / q, b d / q, c d / q ; q)_{\infty}}{\left(a \alpha, a / \alpha, b \alpha, b / \alpha, c \alpha, c / \alpha, d \alpha, d / \alpha, a b c d / q^{3} ; q\right)_{\infty}} J_{\alpha}
$$

where

$$
J_{\alpha}:=\lim _{\substack{n_{i} \rightarrow \infty \\ i=1,2,3,4}} I\left(a q^{n_{1}}, b q^{n_{2}}, c q^{n_{3}}, d q^{n_{4}}\right)=\sum_{k=-\infty}^{\infty} \frac{1-\alpha^{2} q^{2 k}}{1-\alpha^{2}} q^{2 k^{2}}\left(\alpha^{4} / q\right)^{k} .
$$

We may compute $J_{\alpha}$ by using Jacobi's triple product identity [6, II.28] or another formula [6, Ex. 5.5] similar to it but we prefer to take advantage of the fact that $J_{\alpha}$ is independent of $a, b, c, d$. So by setting $d=q / \alpha$ and $c=\alpha q$ in (2.16) and observing that $I_{\alpha}(a, b, \alpha q, q / \alpha)=1$, we get

$$
\begin{aligned}
J_{\alpha} & =\frac{\left(a \alpha, a / \alpha, b \alpha, b / \alpha, q \alpha^{2}, q, q, q / \alpha^{2}, a b / q ; q\right)_{\infty}}{(a b / q, a \alpha, a / \alpha, b \alpha, b / \alpha, q ; q)_{\infty}} \\
& =\left(q \alpha^{2}, q / \alpha^{2}, q ; q\right)_{\infty} .
\end{aligned}
$$


Substituting this into (2.16), we find that

$$
\begin{array}{r}
{ }_{6} \psi_{6}\left[\begin{array}{c}
q \alpha,-q \alpha, \alpha q / a, \alpha q / b, \alpha q / c, \alpha q / d \\
\left.\alpha,-\alpha, \alpha a, \alpha b, \alpha c, \alpha d ; q, a b c d / q^{3}\right]
\end{array}\right. \\
=\frac{\left(q, q \alpha^{2}, q / \alpha^{2}, a b / q, a c / q, a d / q, b c / q, b d / q, c d / q ; q\right)_{\infty}}{\left(\alpha a, \alpha b, \alpha c, \alpha d, a / \alpha, b / \alpha, c / \alpha, d / \alpha, a b c d / q^{3} ; q\right)_{\infty}}
\end{array}
$$

which is the well-known ${ }_{6} \psi_{6}$ summation formula [6, II.33]. Since both sides are analytic if $\operatorname{Im} \alpha \neq 0$ and $\left|a b c d / q^{3}\right|<1$, the restrictive condition that we needed for the validity of (2.9)-(2.11) now can be removed by analytic continuation.

Setting $d=q / \alpha$ in (2.18), we deduce the $q$-Dougall sum [6, II.20]

$$
{ }_{6} \phi_{5}\left[\begin{array}{c}
\alpha^{2}, q \alpha,-q \alpha, \alpha q / a, \alpha q / b, \alpha q / c \\
\alpha,-\alpha, \alpha a, \alpha b, \alpha c
\end{array} ;, a b c / \alpha q^{2}\right]=\frac{\left(q \alpha^{2}, a b / q, a c / q, b c / q ; q\right)_{\infty}}{\left(\alpha a, \alpha b, \alpha c, a b c / \alpha q^{2} ; q\right)_{\infty}}
$$

$\left|a b c / \alpha q^{2}\right|<1$; see also Rogers [15]. Note that, by this method, deriving the ${ }_{6} \psi_{6}$ sum is just as easy as the ${ }_{6} \phi_{5}$ sum.

The Ramanujan- and Barnes-type integrals corresponding to (2.18) had been worked out elsewhere, so we will just state the results. It was Askey [2] who first gave the Ramanujan-type integral

$$
\begin{aligned}
\int_{-\infty}^{\infty} \frac{\left(i a q^{s},-i a q^{-s}, i b q^{s},-i b q^{-s}, i c q^{s},-i c q^{-s}, i d q^{s},-i d q^{-s} ; q\right)_{\infty}}{\left(-q^{1+2 s},-q^{1-2 s} ; q\right)_{\infty}} d s \\
\quad=(q, a b / q, a c / q, a d / q, b c / q, b d / q, c d / q ; q)_{\infty} /\left(a b c d / q^{3} ; q\right)_{\infty},
\end{aligned}
$$

$\left|a b c d / q^{3}\right|<1$. By observing that, under suitable conditions,

$$
\int_{-\infty}^{\infty} f(s) d s=\int_{0}^{1} \sum_{n=-\infty}^{\infty} f(s+n) d s
$$

a slightly more general formula was proved by Ismail and Rahman [8]. Ismail and Masson [7] also found a similar result by an entirely different method.

The Barnes-type integral that corresponds to the ${ }_{6} \psi_{6}$ sum is the well-known AskeyWilson integral

$$
\begin{aligned}
\int_{-\pi / \log \left(q^{-1}\right)}^{\pi / \log \left(q^{-1}\right)} \frac{\left(q^{2 i s}, q^{-2 i s} ; q\right)_{\infty} d s}{\left(a q^{i s}, a q^{-i s}, b q^{i s}, b q^{-i s}, c q^{i s}, c q^{-i s}, d q^{i s}, d q^{-i s} ; q\right)_{\infty}} \\
=\frac{\pi}{\log \left(q^{-1}\right)} \frac{(a b c d ; q)_{\infty}}{(a b, a c, a d, b c, b d, c d, q ; q)_{\infty}}
\end{aligned}
$$

that has been proved by a number of authors since the original proof was published by Askey and Wilson [3] in 1985; see [6] for references. The proof by Atakishiyev and Suslov [5] follows the same path as we have followed in this paper. So, instead of trying to give yet another proof of (2.22), we will go over the first few steps to indicate how one handles such integrals.

The first thing to do is to replace $a, b, c, d$ in (2.1) by $q / a, q / b, q / c, q / d$, respectively, and rewrite $(2.2)$ in the form

$$
\frac{\rho(s+1)}{\rho(s)}=q^{-4 s-2} \frac{\left(1-a q^{s}\right)\left(1-b q^{s}\right)\left(1-c q^{s}\right)\left(1-d q^{s}\right)}{\left(1-a q^{-s-1}\right)\left(1-b q^{-s-1}\right)\left(1-c q^{-s-1}\right)\left(1-d q^{-s-1}\right)}
$$


with general solution in the form

$$
\rho(s)=\frac{q^{-2 s^{2}} \omega(s)}{\left(a q^{s}, a q^{-s}, b q^{s}, b q^{-s}, c q^{s}, c q^{-s}, d q^{s}, d q^{-s} ; q\right)_{\infty}}
$$

where, once again, $\omega(s)$ is a unit-periodic function. The form of this function presented itself in a rather obvious way in (2.6), as well as the Ramanujan integral evaluated in [8]. But the situation is a bit more subtle here. In order to deduce a two-term recurrence relation from (1.1), we need to establish the existence of a contour $C$, parallel to the imaginary axis, such that $\int_{C} \Delta[\rho(s) \sigma(s)] d s=0$. This is satisfied if we require $\max (|a|,|b|,|c|,|d|)<1$. However, we also need a principal periodicity rectangle, symmetrically located about the real axis, in order to obtain a compact integral. This means that we cannot have $q^{-2 s^{2}}$ in the expression (2.24) for $\rho(s)$. So we have to go back to (2.23). Observe that

$$
q^{-4 s-2}=\frac{\left(1-q^{-2 s-1}\right)\left(1-q^{-2 s}\right)}{\left(1-q^{2 s}\right)\left(1-q^{2 s+1}\right)} q^{-1}
$$

and so

$$
\frac{\rho(s+1)}{\rho(s)}=\frac{\left(1-q^{-2 s-1}\right)\left(1-q^{-2 s}\right)\left(1-a q^{s}\right) \cdots\left(1-d q^{s}\right)}{\left(1-q^{2 s+1}\right)\left(1-q^{2 s}\right)\left(1-a q^{-s-1}\right) \cdots\left(1-d q^{-s-1}\right)} q^{-1},
$$

with solution that is appropriate for our purposes given by

$$
\rho(s)=q^{-s} \frac{\left(q^{2 s}, q^{1-2 s} ; q\right)_{\infty}}{\left(a q^{s}, a q^{-s}, \ldots, d q^{s}, d q^{-s} ; q\right)_{\infty}} .
$$

By an argument based on Liouville's theorem, similar to the one used in Section 4 in [11], we can establish the uniqueness of this choice of periodic factor. The next step is to derive a formula similar to (2.5) and set up a 2-term recurrence relation in one of the parameters $a, b, c, d$. For details of the proof, see [5].

3. The $q$-quadratic lattice $x(s)=\frac{1}{2}\left(q^{-s}+q^{s}\right)$ : the ${ }_{8} \psi_{8}$ formula

We now take

$$
\sigma(s)=q^{-2 s} \frac{\left(1-a q^{s-1}\right)\left(1-b q^{s-1}\right)\left(1-c q^{s-1}\right)\left(1-d q^{s-1}\right)\left(1-e q^{s-1}\right)}{\left(1-f q^{s-1}\right)}
$$

where $a, b, c, d, e, f$ are complex parameters satisfying the balance condition

$$
a b c d e=f q^{4} .
$$

This leads to the following solution of (1.1):

$$
\rho(s)=\frac{\left(a q^{-s}, a q^{s}, \ldots, e q^{-s}, e q^{s} ; q\right)_{\infty}}{\left(f q^{-s}, f q^{s} ; q\right)_{\infty}} q^{2 s^{2}} \omega(s), \quad \omega(s \pm 1)=\omega(s) .
$$


Hence,

$$
\begin{aligned}
\tau(s) \nabla x_{1}(s)= & \sigma(-s)-\sigma(s) \\
= & {\left[-\frac{\left(1-a b / q^{2}\right)\left(1-a c / q^{2}\right)\left(1-a d / q^{2}\right)\left(1-a e / q^{2}\right)}{\left(1-a f / q^{2}\right)}\right.} \\
& \left.+\frac{(1-f / b)(1-f / c)(1-f / d)(1-f / e)}{\left(1-a f / q^{2}\right)} \frac{\left(1-a q^{s-1}\right)\left(1-a q^{-s-1}\right)}{\left(1-f q^{s-1}\right)\left(1-f q^{-s-1}\right)}\right] \\
& \quad \times \frac{q\left(q^{-s}-q^{s}\right)}{a},
\end{aligned}
$$

which, on replacing $\rho(s)$ by $\rho(s ; a)$, gives

$$
\begin{aligned}
& \frac{a}{q} \Delta[\rho(s ; a) \sigma(s)] \\
& =-\frac{\left(1-a b / q^{2}\right)\left(1-a c / q^{2}\right)\left(1-a d / q^{2}\right)\left(1-a e / q^{2}\right)}{1-a f / q^{2}} \rho(s ; a)\left(q^{-s}-q^{s}\right) \\
& \quad+\frac{(1-f / b)(1-f / c)(1-f / d)(1-f / e)}{1-a f / q^{2}} \rho\left(s ; a q^{-1}\right)\left(q^{-s}-q^{s}\right) .
\end{aligned}
$$

Note that, by (3.2), as $a$ goes to $a / q, f$ must go to $f / q$. Similar to Section 2 , let $s_{0} \in \mathbf{C}$, such that $\operatorname{Im} s_{0} \neq 0$, and take

$$
\omega\left(s_{0}\right)=\frac{q^{s_{0}-2 s_{0}^{2}}\left(f q^{-s_{0}}, f q^{s_{0}} ; q\right)_{\infty}}{\left(1-q^{2 s_{0}}\right)\left(a q^{-s_{0}}, a q^{s_{0}}, \ldots, e q^{-s_{0}}, e q^{s_{0}} ; q\right)_{\infty}} .
$$

Denote $q^{s_{0}}=\alpha$, and

$$
\begin{aligned}
I_{\alpha}(a, b, c, d, e) & =\sum_{k=-\infty}^{\infty} \frac{(\alpha q / a, \alpha q / b, \alpha q / c, \alpha q / d, \alpha q / e, \alpha f ; q)_{k}}{(\alpha a, \alpha b, \alpha c, \alpha d, \alpha e, \alpha q / f ; q)_{k}} \frac{\left(1-\alpha^{2} q^{2 k}\right)}{1-\alpha^{2}} q^{k} \\
& ={ }_{8} \psi_{8}\left[\begin{array}{c}
q \alpha,-q \alpha, \alpha q / a, \alpha q / b, \alpha q / c, \alpha q / d, \alpha q / e, \alpha f \\
\alpha,-\alpha, \alpha a, \alpha b, \alpha c, \alpha d, \alpha e, \alpha q / f
\end{array} ;, q\right] .
\end{aligned}
$$

This very-well-poised bilateral series also is balanced because of (3.2) and so converges whenever $|q|<1$. Summing the relation (3.5) from $s=s_{0}-\ell$ to $s=s_{0}+k$, $k=0,1,2, \ldots, \ell=1,2, \ldots$, and taking the limits $k \rightarrow \infty, \ell \rightarrow \infty$, we get

$$
\begin{aligned}
& \frac{(1-f / b)(1-f / c)(1-f / d)(1-f / e)(1-a \alpha / q)(1-a / \alpha q)}{\left(1-a f / q^{2}\right)(1-f \alpha / q)(1-f / \alpha q)} I_{\alpha}\left(a q^{-1}, b, c, d, e\right) \\
& -\frac{\left(1-a b / q^{2}\right)\left(1-a c / q^{2}\right)\left(1-a d / q^{2}\right)\left(1-a e / q^{2}\right)}{\left(1-a f / q^{2}\right)} I_{\alpha}(a, b, c, d, e) \\
& =\frac{a}{q \omega\left(s_{0}\right)}\left[\lim _{k \rightarrow \infty} \rho\left(s_{0}+k\right) \sigma\left(s_{0}+k\right)-\lim _{\ell \rightarrow \infty} \rho\left(s_{0}-\ell\right) \sigma\left(s_{0}-\ell\right)\right] .
\end{aligned}
$$

Now, by (3.1)-(3.3) and (3.6),

$$
\omega^{-1}\left(s_{0}\right) \lim _{k \rightarrow \infty} \rho\left(s_{0}+k\right) \sigma\left(s_{0}+k\right)=\frac{(\alpha q / a, \ldots, \alpha q / e, \alpha f ; q)_{\infty}}{\alpha\left(1-\alpha^{2}\right)(\alpha a, \ldots, \alpha e, \alpha q / f ; q)_{\infty}}
$$

and

$$
\omega^{-1}\left(s_{0}\right) \lim _{\ell \rightarrow \infty} \rho\left(s_{0}-\ell\right) \sigma\left(s_{0}-\ell\right)=\frac{\alpha^{3}}{1-\alpha^{2}} \frac{(q / \alpha a, \ldots, q / \alpha e, f / \alpha ; q)_{\infty}}{(a / \alpha, \ldots, e / \alpha, q / \alpha f ; q)_{\infty}} .
$$


Replacing $a$ by $q a$ (and so $f$ by $f q$ ), we obtain a nonhomogeneous recurrence relation

$$
\begin{aligned}
& I_{\alpha}(a, b, c, d, e) \\
& =\frac{(1-\alpha f)(1-f / \alpha)(1-a b / q)(1-a c / q)(1-a d / q)(1-a e / q)}{(1-\alpha a)(1-a / \alpha)(1-f q / b)(1-f q / c)(1-f q / d)(1-f q / e)} I_{\alpha}(a q, b, c, d, e) \\
& +\frac{(\alpha q / a, \ldots, \alpha q / e, \alpha f ; q)_{\infty}}{(\alpha a, \ldots, \alpha e, \alpha q / f ; q)_{\infty}} \frac{f(1-a f) p_{\alpha}(a, b, c, d, e)}{(1-f q / b)(1-f q / c)(1-f q / d)(1-f q / e) \alpha\left(1-\alpha^{2}\right)}
\end{aligned}
$$

where

$$
p_{\alpha}(a, b, c, d, e)=1-\alpha^{4} \frac{(\alpha a, \ldots, \alpha e, q / \alpha a, \ldots, q / \alpha e, f / \alpha, \alpha q / f ; q)_{\infty}}{(a / \alpha, \ldots, e / \alpha, \alpha q / a, \ldots, \alpha q / e, \alpha f, q / \alpha f ; q)_{\infty}} .
$$

It is obvious that $p_{\alpha}(a, b, c, d, e)$ is symmetric in $a, b, c, d, e$. Also, it can be easily verified that it has a periodic property in all of these parameters, e.g.,

$$
p_{\alpha}(a q, b, c, d, e)=p_{\alpha}(a, b, c, d, e) .
$$

Iterating (3.11) once, we get

$$
\begin{aligned}
I_{\alpha}(a, b, c, d, e)= & \frac{(\alpha f, f / \alpha, a b / q, a c / q, a d / q, a e / q ; q)_{2}}{(\alpha a, a / \alpha, f q / b, f q / c, f q / d, f q / e ; q)_{2}} I_{\alpha}\left(a q^{2}, b, c, d, e\right) \\
+ & \frac{(\alpha q / a, \ldots, \alpha q / e, \alpha f ; q)_{\infty}}{(\alpha a, \ldots, \alpha e, \alpha q / f ; q)_{\infty}} \\
& \times \frac{f(1-a f) p_{\alpha}(a, b, c, d, e)}{(1-f q / b)(1-f q / c)(1-f q / d)(1-f q / e) \alpha\left(1-\alpha^{2}\right)} \\
& \times \sum_{k=0}^{1} \frac{\left(1-a f q^{2 k}\right)(a b / q, a c / q, a d / q, a e / q ; q)_{k}}{(1-a f)\left(f q^{2} / b, f q^{2} / c, f q^{2} / d, f q^{2} / e ; q\right)_{k}}(f q / a)^{k} .
\end{aligned}
$$

By induction, we can prove that

$$
\begin{aligned}
& I_{\alpha}(a, b, c, d, e)=\frac{(\alpha f, f / \alpha, a b / q, a c / q, a d / q, a e / q ; q)_{n}}{(\alpha a, a / \alpha, f q / b, f q / c, f q / d, f q / e ; q)_{n}} I_{\alpha}\left(a q^{n}, b, c, d, e\right) \\
& +\frac{(\alpha q / a, \ldots, \alpha q / e, \alpha f ; q)_{\infty}}{(\alpha a, \ldots, \alpha e, \alpha q / f ; q)_{\infty}} \\
& \quad \times \frac{f(1-a f) p_{\alpha}(a, b, c, d, e)}{\alpha\left(1-\alpha^{2}\right)(1-f q / b)(1-f q / c)(1-f q / d)(1-f q / e)} \\
& \quad \times \sum_{k=0}^{n-1} \frac{\left(1-a f q^{2 k}\right)(a b / q, a c / q, a d / q, a e / q ; q)_{k}}{(1-a f)\left(f q^{2} / b, f q^{2} / c, f q^{2} / d, f q^{2} / e ; q\right)_{k}}(f q / a)^{k} .
\end{aligned}
$$

From (3.7), we find that

$$
\begin{aligned}
& \lim _{n \rightarrow \infty} I_{\alpha}\left(a q^{n}, b, c, d, e\right) \\
& =\lim _{n \rightarrow \infty}{ }_{8} \psi_{8}\left[\begin{array}{c}
q \alpha,-q \alpha, \alpha q^{1-n} / a, \alpha q / b, \alpha q / c, \alpha q / d, \alpha q / e, \alpha f q^{n} \\
\alpha,-\alpha, \alpha a q^{n}, \alpha b, \alpha c, \alpha d, \alpha e, \alpha q^{1-n} / f
\end{array} ; q, q\right] \\
& ={ }_{6} \psi_{6}\left[\begin{array}{c}
q \alpha,-q \alpha, \alpha q / b, \alpha q / c, \alpha q / d, \alpha q / e \\
\alpha,-\alpha, \alpha b, \alpha c, \alpha d, \alpha e
\end{array} ; q, f q / a\right] \\
& =\frac{\left(q, q \alpha^{2}, q / \alpha^{2}, b c / q, b d / q, b e / q, c d / q, c e / q, d e / q ; q\right)_{\infty}}{(\alpha b, \alpha c, \alpha d, \alpha e, b / \alpha, c / \alpha, d / \alpha, e / \alpha, f q / a ; q)_{\infty}}
\end{aligned}
$$


by $(2.18)$, provided

$$
|f q / a|=\left|b c d e / q^{3}\right|<1
$$

Combining (3.15) with the $n \rightarrow \infty$ limit of (3.14), we obtain

$$
\begin{aligned}
& I_{\alpha}(a, b, c, d, e) \\
& \begin{array}{l}
=\frac{\left(q, q \alpha^{2}, q / \alpha^{2}, \alpha f, f / \alpha, a b / q, a c / q, a d / q, a e / q, b c / q, b d / q, b e / q, c d / q, c e / q, d e / q ; q\right)_{\infty}}{(\alpha a, \alpha b, \alpha c, \alpha d, \alpha e, a / \alpha, b / \alpha, c / \alpha, d / \alpha, e / \alpha, f q / a, f q / b, f q / c, f q / d, f q / e ; q)_{\infty}} \\
\quad+\frac{(\alpha q / a, \ldots, \alpha q / e, \alpha f ; q)_{\infty}}{(\alpha a, \ldots, \alpha e, \alpha q / f ; q)_{\infty}} \frac{f(1-a f) p_{\alpha}(a, b, c, d, e)}{\alpha\left(1-\alpha^{2}\right)(1-f q / b)(1-f q / c)(1-f q / d)(1-f q / e)} \\
\quad \times{ }_{8} \phi_{7}\left[\begin{array}{l}
a f, q \sqrt{a f},-q \sqrt{a f}, a b / q, a c / q, a d / q, a e / q, q \\
\sqrt{a f},-\sqrt{a f}, f q^{2} / b, f q^{2} / c, f q^{2} / d, f q^{2} / e, a f
\end{array} ;, f q / a\right] .
\end{array}
\end{aligned}
$$

Observe that setting $\alpha=f$, we obtain a transformation formula

$$
\begin{gathered}
{ }_{8} \phi_{7}\left[\begin{array}{c}
\left.f^{2}, q f,-q f, f q / a, f q / b, f q / c, f q / d, f q / e ; q, q\right] \\
f,-f, a f, b f, c f, d f, e f
\end{array}\right] \\
=\frac{\left(f q / a, f q^{2} / b, f q^{2} / c, f q^{2} / d, f q^{2} / e, q f^{2} ; q\right)_{\infty}}{(a f q, b f, c f, d f, e f, q ; q)_{\infty}} \\
\quad \times{ }_{8} \phi_{7}\left[\begin{array}{l}
a f, q \sqrt{a f},-q \sqrt{a f}, a b / q, a c / q, a d / q, a e / q, q \\
\sqrt{a f},-\sqrt{a f}, f q^{2} / b, f q^{2} / c, f q^{2} / d, f q^{2} / e, a f
\end{array} ; q, f q / a\right]
\end{gathered},
$$

which is a special case of Bailey's transformation formula [6, III.24]. Substituting (3.18) into (3.17), we find that

$$
\begin{aligned}
{ }_{8} \psi_{8}\left[\begin{array}{c}
q \alpha,-q \alpha, \alpha q / a, \alpha q / b, \alpha q / c, \alpha q / d, \alpha q / e, \alpha f \\
\alpha,-\alpha, \alpha a, \alpha b, \alpha c, \alpha d, \alpha e, \alpha q / f
\end{array} ;, q\right] \\
=\frac{\left(q, q \alpha^{2}, q / \alpha^{2}, \alpha f, f / \alpha, a b / q, a c / q, a d / q, a e / q, b c / q, b d / q, b e / q, c d / q, c e / q, d e / q ; q\right)_{\infty}}{(\alpha a, \alpha b, \alpha c, \alpha d, \alpha e, a / \alpha, b / \alpha, c / \alpha, d / \alpha, e / \alpha, f q / a, f q / b, f q / c, f q / d, f q / e ; q)_{\infty}} \\
+\frac{f p_{\alpha}(a, b, c, d, e)}{\alpha\left(1-\alpha^{2}\right)} \frac{(q, \alpha q / a, \alpha q / b, \alpha q / c, \alpha q / d, \alpha q / e, \alpha f, a f, b f, c f, d f, e f ; q)_{\infty}}{\left(\alpha a, \alpha b, \alpha c, \alpha d, \alpha e, \alpha q / f, f q / a, f q / b, f q / c, f q / d, f q / e, q f^{2} ; q\right)_{\infty}} \\
\quad \times{ }_{8} \phi_{7}\left[\begin{array}{c}
f^{2}, q f,-q f, f q / a, f q / b, f q / c, f q / d, f q / e \\
f,-f, a f, b f, c f, d f, e f
\end{array}\right],
\end{aligned}
$$

provided $\max (|f q / a|,|f q / b|,|f q / c|,|f q / d|,|f q / e|)<1$.

If $\alpha e=q$, then $p_{\alpha}(a, b, c, d, e)=1$, and we obtain

$$
\begin{array}{r}
{ }_{8} \phi_{7}\left[\begin{array}{c}
q^{2} / e^{2}, q^{2} / e,-q^{2} / e, q^{2} / a e, q^{2} / b e, q^{2} / c e, q^{2} / d e, q f / e \\
q / e,-q / e, a q / e, b q / e, c q / e, d q / e, q^{2} / e f
\end{array} ;, q\right] \\
+\frac{\left(e f / q, a f, b f, c f, d f, q^{3} / e^{2}, q^{2} / a e, q^{2} / b e, q^{2} / c e, q^{2} / d e ; q\right)_{\infty}}{\left(q / e f, f q / a, f q / b, f q / c, f q / d, q f^{2}, a q / e, b q / e, c q / e, d q / e ; q\right)_{\infty}} \\
\quad \times{ }_{8} \phi_{7}\left[\begin{array}{c}
f^{2}, q f,-q f, f q / a, f q / b, f q / c, f q / d, f q / e \\
f,-f, a f, b f, c f, d f, e f
\end{array}\right] \\
=\frac{\left(q^{3} / e^{2}, a b / q, a c / q, a d / q, b c / q, b d / q, c d / q, e f / q ; q\right)_{\infty}}{(a q / e, b q / e, c q / e, d q / e, f q / a, f q / b, f q / c, f q / d ; q)_{\infty}},
\end{array}
$$

which is the nonterminating $q$-Jackson formula due to Bailey [6, II.25]. Finally, if $\alpha$, $a, b, c, d, e, f$ satisfy the relation

$$
p_{\alpha}(a, b, c, d, e)=0
$$


then we have Gosper's summation formula [6, Ex. 5.12] for a special ${ }_{8} \psi_{8}$ series.

The proof of (3.19) given here is the same as that in [13, §8], except that we have been able to express the formula in a symmetric form in all five parameters $a, b, c$, $d, e$, which enabled us to derive (3.20) without having to borrow any transformation formulas. Furthermore, the fact that $p_{\alpha}(a, b, c, d, e)$ emerges from the asymptotic values of $\rho(s) \sigma(s)$ from the two ends of the real line has been made more clear here than we were able to do in [13].

The Barnes-type integral that corresponds to the ${ }_{8} \psi_{8}$ sum also was worked out in [13], so we shall just state the results:

$$
\begin{array}{r}
\int_{-i \pi / \log \left(q^{-1}\right)}^{i \pi / \log \left(q^{-1}\right)} \frac{\left(q^{2 s}, q^{-2 s}, f q^{s}, f q^{-s} ; q\right)_{\infty} d s}{\left(a q^{s}, a q^{-s}, b q^{s}, b q^{-s}, c q^{s}, c q^{-s}, d q^{s}, d q^{-s}, e q^{s}, e q^{-s} ; q\right)_{\infty}} \\
=\frac{\pi i}{\log \left(q^{-1}\right)} \frac{(f / a, f / b, f / c, f / d, f / e ; q)_{\infty}}{(q, a b, a c, a d, a e, b c, b d, b e, c d, c e, d e ; q)_{\infty}}
\end{array}
$$

where $f=a b c d e$ and $\max (|a|,|b|,|c|,|d|,|e|)<1$. This is better known as the Nassrallah-Rahman formula [9]. An alternate proof also was given by Askey [1], which is pretty close to the proof in [13].

The Ramanujan-type integral that may be thought of as a direct integral analogue of (3.19) also was worked out in [13] by this Pearson-equation technique, and in [8] by the use of (2.21). In [8], however, some transformation formulas were used for which there is really no need. So, let us sketch another short proof based on (3.19) only. As an extension of the integral in (2.20), let us consider

$$
K_{\alpha}(a, b, c, d, e)=\int_{-\infty}^{\infty} \frac{\left(\alpha a q^{s}, a q^{-s} / \alpha, \ldots, \alpha e q^{s}, e q^{-s} / \alpha ; q\right)_{\infty}}{\left(\alpha^{2} q^{2 s+1}, q^{1-2 s} / \alpha^{2}, \alpha f q^{s}, f q^{-s} / \alpha ; q\right)_{\infty}} d s
$$

where $a, b, c, d, e, f$ satisfy the balance condition (3.2); $\alpha$ can be quite arbitrary, including 1 or any integer power of $q$ (in which case the integrand will have simple poles on the real line and then the integral has to be interpreted in a principal value sense; see [13]). To avoid unnecessary complications, we shall just assume that $\operatorname{Im} \alpha \neq 0$. By (2.21), we then have

$$
\begin{aligned}
K_{\alpha}(a, b, c, d, e)= & \int_{0}^{1} \frac{\left(\alpha a q^{s}, a q^{-s} / \alpha, \ldots, \alpha e q^{s}, e q^{-s} / \alpha ; q\right)_{\infty}}{\left(\alpha^{2} q^{2 s+1}, q^{1-2 s} / \alpha^{2}, \alpha f q^{s}, f q^{-s} / \alpha ; q\right)_{\infty}} \\
& \times{ }_{8} \psi_{8}\left[\begin{array}{c}
\alpha q^{s+1},-\alpha q^{s+1}, \alpha q^{s+1} / a, \ldots, \alpha q^{s+1} / e, \alpha f q^{s} \\
\alpha q^{s},-\alpha q^{s}, \alpha a q^{s}, \ldots, \alpha e q^{s}, \alpha q^{s+1} / f
\end{array} ; q, q\right] d s .
\end{aligned}
$$

By (3.19), this ${ }_{8} \psi_{8}$ series equals

$$
\begin{aligned}
& \frac{\left(q, \alpha^{2} q^{1+2 s}, q^{1-2 s} / \alpha^{2}, \alpha f q^{s}, f q^{-s} / \alpha ; q\right)_{\infty}}{\left(\alpha a q^{s}, a q^{-s} / \alpha, \ldots, \alpha e q^{s}, e q^{-s} / \alpha ; q\right)_{\infty}} \frac{(a b / q, a c / q, \ldots, c e / q, d e / q ; q)_{\infty}}{(f q / a, \ldots, f q / e ; q)_{\infty}} \\
& \quad+\frac{f(q, a f, b f, c f, d f, e f ; q)_{\infty}}{\left(f q / a, f q / b, f q / c, f q / d, f q / e, q f^{2} ; q\right)_{\infty}}{ }_{8} \phi_{7}\left[\begin{array}{c}
f^{2}, q f,-q f, f q / a, \ldots, f q / e \\
f,-f, a f, \ldots, e f
\end{array} ; q, q\right] \\
& \quad \times \frac{\left(\alpha q^{s+1} / a, \ldots, \alpha q^{s+1} / e, \alpha f q^{s} ; q\right)_{\infty}}{\left(\alpha a q^{s}, \ldots, \alpha e q^{s}, \alpha q^{s+1} / f ; q\right)_{\infty}} \frac{p_{\alpha q^{s}}(a, b, c, d, e)}{\alpha q^{s}\left(1-\alpha^{2} q^{2 s}\right)}
\end{aligned}
$$


Substituting this into (3.24) yields the formula

$$
\begin{aligned}
& \int_{-\infty}^{\infty} \frac{\left(\alpha a q^{s}, a q^{-s} / \alpha, \ldots, \alpha e q^{s}, e q^{-s} / \alpha ; q\right)_{\infty}}{\left(\alpha^{2} q^{1+2 s}, q^{1-2 s} / \alpha^{2}, \alpha f q^{s}, f q^{-s} / \alpha ; q\right)_{\infty}} d s \\
& =\frac{(q, a b / q, a c / q, a d / q, a e / q, b c / q, b d / q, c d / q, c e / q, d e / q ; q)_{\infty}}{(f q / a, f q / b, f q / c, f q / d, f q / e ; q)_{\infty}} \\
& \quad+\frac{f(a f, b f, c f, d f, e f, q ; q)_{\infty}}{\alpha\left(f q / a, f q / b, f q / c, f q / d, f q / e, q f^{2} ; q\right)_{\infty}} \\
& \quad \times{ }_{8} \phi_{7}\left[\begin{array}{c}
f^{2}, q f,-q f, f q / a, f q / b, \ldots, f q / e \\
f,-f, a f, b f, \ldots, e f
\end{array}\right] \\
& \times \int_{0}^{1} q^{-s} \frac{\left(\alpha q^{s+1} / a, a q^{-s} / \alpha, \ldots, \alpha q^{s+1} / e, e q^{-s} / \alpha ; q\right)_{\infty}}{\left(\alpha^{2} q^{2 s}, q^{1-2 s} / \alpha^{2}, \alpha q^{s+1} / f, f q^{-s} / \alpha ; q\right)_{\infty}} \\
& \quad \times\left[1-\alpha^{4} q^{4 s} \frac{\left(\alpha a q^{s}, q^{1-s} / a \alpha, \ldots, e \alpha q^{s}, q^{1-s} / e \alpha, f q^{-s} / \alpha, \alpha q^{s+1} / f ; q\right)_{\infty}}{\left(a q^{-s} / \alpha, \alpha q^{s+1} / a, \ldots, e q^{-s} / \alpha, \alpha q^{s+1} / e, \alpha f q^{s}, q^{1-s} / \alpha f ; q\right)_{\infty}}\right] d s .
\end{aligned}
$$

In this case, there doesn't seem to be a Gosper-type formula.

Acknowledgment. This work, supported in part by the NSERC grant \#A6197, was completed while the second author was visiting Carleton University in SeptemberOctober, 1993.

\section{References}

1. R. Askey, Beta integrals in Ramanujan's papers, his unpublished work and further examples. In: Ramanujan Revisited (G. E. Andrews et al., eds.), Academic Press, New York, 1988, pp.561-590.

2. , Beta integrals and q-extensions. In: Proceedings of the Ramanujan Centennial International Conference, Annamalainagar, (R. Balakrishnan et al., eds.), Ramanujan Mathematical Society, Annamalainagar University, 1988, pp.85-102.

3. R. Askey and J. A. Wilson, Some basic hypergeometric polynomials that generalize Jacobi polynomials, Mem. Amer. Math. Soc. 319, 1985.

4. N. M. Atakishiyev, M. Rahman, and S. K. Suslov, On the classical orthogonal polynomials, Constr. Approx. 11 (1995), 181-226.

5. N. M. Atakishiyev and S. K. Suslov, On the Askey-Wilson polynomials, Constr. Approx. 8 (1992), 363-369.

6. G. Gasper and M. Rahman, Basic Hypergeometric Series, Cambridge University Press, Cambridge, 1990.

7. M. E. H. Ismail and D. Masson, q-Hermite polynomials, biorthogonal rational functions, and $q$-beta integrals, Trans. Amer. Math. Soc. 346 (1994), 63-116.

8. M. E. H. Ismail and M. Rahman, Some basic bilateral series and integrals, Pacific J. Math. 170 (1995), 497-515.

9. B. Nassrallah and M. Rahman, Projection formulas, a reproducing kernel and a generating function for $q$-Wilson polynomials, SIAM J. Math. Anal. 16 (1985), 186-197.

10. A. F. Nikiforov, S. K. Suslov, and V. B. Uvarov, Classical Orthogonal Polynomials of a Discrete Variable, Nauka, Moscow, 1985 (in Russian); English translation, Springer-Verlag, 1991.

11. M. Rahman and S. K. Suslov, (1996). A unified approach to the summation and integration formulas for q-hypergeometric functions I, Journal of Statistical Planning and Inference 54 (1996), 101-118.

12. $-A$ unified approach to the summation and integration formulas for q-hypergeometric functions II, Methods and Application Analysis 5 (1998), xxx-xxx.

13. The Pearson equation and the beta integrals, SIAM J. Math. Anal. 25 (1994), 646-693.

14. , Barnes and Ramanujan-type integrals on the q-linear lattice, SIAM J. Math. Anal. 25 (1994), 1002-1022. 
15. L. J. Rogers, Third memoir on the expansion of certain infinite products, Proc. London Math. Soc. 26 (1895), 15-32.

16. S. K. Suslov, The theory of difference analogues of special functions of hypergeometric type, Russian Mathematical Surveys, 44 (1989), London Math. Soc. (in English) (1989), 227-278.

17. —_ Multiparameter Ramanujan-type q-beta integrals, The Ramanujan Journal 2 (1998), $30-49$.

School of Mathematics and Statistics, Carleton University, 1125 Colonel By Drive, OttaWA, Ontario, Canada K1S 5B6

Russian Research Centre, Kurchatov Institute, Moscow 123182, Russia, and Department of Mathematics, Arizona State University, Tempe, Arizona 85287-1804, U.S.A.

E-mail: suslov@math.la.asu.edu 